\title{
A minimal mathematical model of neutrophil pseudopodium formation during chemotaxis
}

\author{
by J.J.D. Korobkin ${ }^{1}$, A. Proensa-Garcia ${ }^{2}$, A.N. Sveshnikova ${ }^{1-4} \#$
}

1. Center for Theoretical Problems of Physico-Chemical Pharmacology, Russian Academy of Sciences, 30 Srednyaya Kalitnikovskaya str., Moscow, 109029, Russia

2. Faculty of Physics, Lomonosov Moscow State University, 1/2 Leninskie gory, Moscow, 119991, Russia

3. National Medical Research Center of Pediatric Hematology, Oncology and Immunology named after Dmitry Rogachev, 1 Samory Mashela St, Moscow, 117198, Russia

4. Department of Normal Physiology, Sechenov First Moscow State Medical University, 8/2 Trubetskaya St., Moscow, 119991 , Russia

\# Correspondence: a.sveshnikova@physics.msu.ru

\section{Abstract}

The directed movement of neutrophils is provided by the rapid polymerization of actin with the formation of a protrusion growing forward. In our previous work we observed impaired neutrophil movement for patients with Wiskott-Aldrich syndrome (WAS) compared to healthy donors.

In this work, we set out to explain the impairment of neutrophil chemotaxis in patients by observation and computer modeling of the linear growth rates of the anterior pseudopodia. The neutrophil chemotaxis was observed by means of low-angle fluorescent microscopy in parallel-plate flow chambers. The computational model was constructed as a networklike 2D stochastic polymerization of actin guided by the proximity of cell membrane with branching governed by Arp2/3 and WASP proteins.

The observed linear velocity of neutrophil pseudopodium formation was $0.22 \pm 0.04 \mu \mathrm{m} / \mathrm{s}$ for healthy donors and $0.23 \pm 0.08 \mu \mathrm{m} / \mathrm{s}$ for WAS patients. The model described the velocity of the pseudopodium formation for healthy donors well. For the description of WAS patients data, a variation of branching velocity (governed by WASP) by an order of magnitude was applied, which did not significantly alter the linear protrusion growth velocity.

We conclude that the proposed mathematical model of neutrophil pseudopodium formation could describe the experimental data well, but the data on overall neutrophil movement could not be explained by the velocities of the pseudopodium growth.

Keywords: Cytoskeleton, neutrophils, actin, chemotaxis, Wiskott-Aldrich syndrome

\section{Introduction}

Neutrophils represent the largest population of leukocytes in human blood and they are the first cells to be recruited to the sites of inflammation [1]. To perform their functions, neutrophils need to detect extracellular chemical gradients and move towards higher concentrations of chemoattractants emanating from the sites of injury. This process is called chemotaxis or guided cell migration [2]. Chemotaxis of neutrophils is characterized by gradient sensitivity, polarization, and cell motility [3]. Chemoattractant sensing occurs through G-protein coupled receptors (GPCRs) and other receptors that induce asymmetric localization and activation of key signaling molecules.

For the directed movement an actin cytoskeleton rearrangement should happen. Specifically, actin polymerization happens on the growing towards the source of chemoattractant cell protrusion. This protrusion is sometimes called pseudopodia (or, in neutrophils, lamellipodia).

The neutrophil pseudopodium consists of a core rich in actin surrounded by adhesion and scaffolding proteins. Globular $(G)$ actin monomers are polymerized to form filamentous (F) actin, which is critical for cell migration [4,5]. F-actin are oriented filaments that grow at the so-called barbed end push the front of the cell forward, which stimulates cell migration. Actin nucleation and polymerization are regulated by formins (e.g., mDia1 and mDia2) and the Arp $2 / 3$ complex $[5,6]$. Formins nucleate regulate the growth of linear actin filaments $[7,8]$. The Arp2/3 complex generates branches from existing actin filaments at an angle of $70^{\circ}$ and thus creates a dendritic actin network [2].

Dynamics of neutrophil movements could be considered as an indicator of neutrophils' adequacy. Recently we have suggested an experimental model for assessing the adequacy of a patients' neutrophils in an ex vivo setting of growing thrombi 
[3]. In that work we looked at the movement of neutrophils of pediatric patients with Wiskott-Aldrich syndrome (WAS) - a rare genetic immunodeficiency characterized by immune dysregulation and cytoskeletal abnormalities. Immune cells from WAS patients display lower chemotaxis velocities $[3,9]$. It is known that the Arp2/3 (Actin-related proteins 2/3) complex is activated by the WASP (Wiskott-Aldrich syndrome protein) family [10], but the influence of WASp mutations on lamellipodia formation is not clear yet.

Mathematical modeling has been applied to describe actin cytoskeleton growth in lamellipodia [11], filopodia [12] and presynaptic membrane protrusions [13]. There are several possible approaches to model the neutrophil pseudopodium formation, such as continuous modeling of actin concentration [14] or stochastic modeling of individual proteins. Since it has been shown that the neutrophil membrane protrusions are intrinsically lamellar [15], a two-dimensional (2D) geometry could be used to describe the membrane protrusion growth.

Here we made a first attempt to bind together the observed changes in neutrophils' movements for WAS patients and the known mechanisms of actin polymerization and neutrophil pseudopodium formation. We measured the linear velocities of the neutrophil pseudopodium growth for WAS patients and healthy donors by means of fluorescent microscopy. Surprisingly, the difference between these values was not significant. To explain the observed phenomena, we have built a minimal mathematical model capable of quantitative description of neutrophil membrane protrusion growth. In accordance with experimental data, varying branching velocity in an order of magnitude did not significantly alter the overall protrusion growth velocity. It indicates that the observed disruption of chemotaxis in WAS patients could not be explained by impaired pseudopodium formation.

\section{Materials and Methods}

\section{Materials}

The sources of the materials were as follows: DiOC-6, bovine serum albumin (BSA), human fibrinogen (Sigma-Aldrich, St Louis, MO); fibrillar collagen type I (Chrono- Log Corporation; Havertown; USA).

\section{Fluorescent microscopy}

Parallel-plate flow chambers were mounted as described previously [16] Channel parameters were: $0.2 \times 18 \times 0.206 \mathrm{~mm}$. Glass coverslips were coated with fibrillar collagen type I $(0.2 \mathrm{mg} / \mathrm{ml})$ for $1 \mathrm{~h} 30 \mathrm{~min}$ at $37^{\circ} \mathrm{C}$, washed with distilled water, blocked with BSA and then inserted into the flow chambers.

Blood was collected from healthy adult volunteers
( $n=5$, men and women 18-35 years old) or from patients with Wiskott-Aldrich syndrome $(n=3)$ into Sarstedt-Monovette $\subset$ hirudin (525 ATU/ml blood) vacuum tubes.

Whole blood was pre-loaded with DiOc6 (1 $\mu \mathrm{M})$. Blood was perfused through the parallel-plate chambers over collagen-coated $(0.2 \mathrm{mg} / \mathrm{ml})$ surface with wall shear rates of $100 \mathrm{~s}^{-1}$ as described in [3]. Thrombus growth and leukocyte crawling were observed in DIC/epifluorescence modes with an inverted Nikon Eclipse Ti-E microscope (100x/1.49 NA TIRF oil objective). Neutrophil locations were derived based on DiOC6 fluorescence. Alternatively, confocal mode with Z1 microscope (Carl Zeiss, Jena, Germany; 100x objective; Axio Observer) was used. $488 \mathrm{~nm}$ laser was applied to assess DiOC6 fluorescence in neutrophils. It has been already shown that the attaching cells in this experimental setup are exclusively neutrophils [3].

\section{Data analysis}

Nikon NIS-Elements software was used for microscope image acquisition; ImageJ [17] (http:// imagej.net/ImageJ) was used for image processing. ImageJ manual tracking plugin was used for manual granulocyte pseudopod tracking.

\section{Statistics}

All experiments were performed at least in triplicate with platelets from different donors. Statistical analysis was performed using Python 3.6; all statistical details are provided in the figure captions.

\section{Results}

Experimental measurement of the linear velocities of pseudopodium formation.

To measure the rate of anterior protrusion formation, a series of experiments were carried out in which neutrophils movements were observed during thrombus growth in whole hirudinated blood (without any external chemoattractant). The measurements were performed only for the moving neutrophils. The linear velocity of the protrusion was measured by the following algorithm: a) the moving cell with one distinct pseudopodium was selected; b) the central line in the direction of the protrusion was identified; c) the coordinates of the growing "tip" along this line were taken by means of ImageJ software; d) the instant and average velocities of the protrusion were calculated from these coordinates (Fig. 1A).

For healthy donors the average protrusion velocities appeared to be $0.22 \pm 0.04 \mu \mathrm{m} / \mathrm{s}$, while for WAS patients the average protrusion velocities were $0.23 \pm 0.08 \mu \mathrm{m} / \mathrm{s}$. According to the Manne-Whitney criterion, the difference between healthy and WAS data was not significant. 

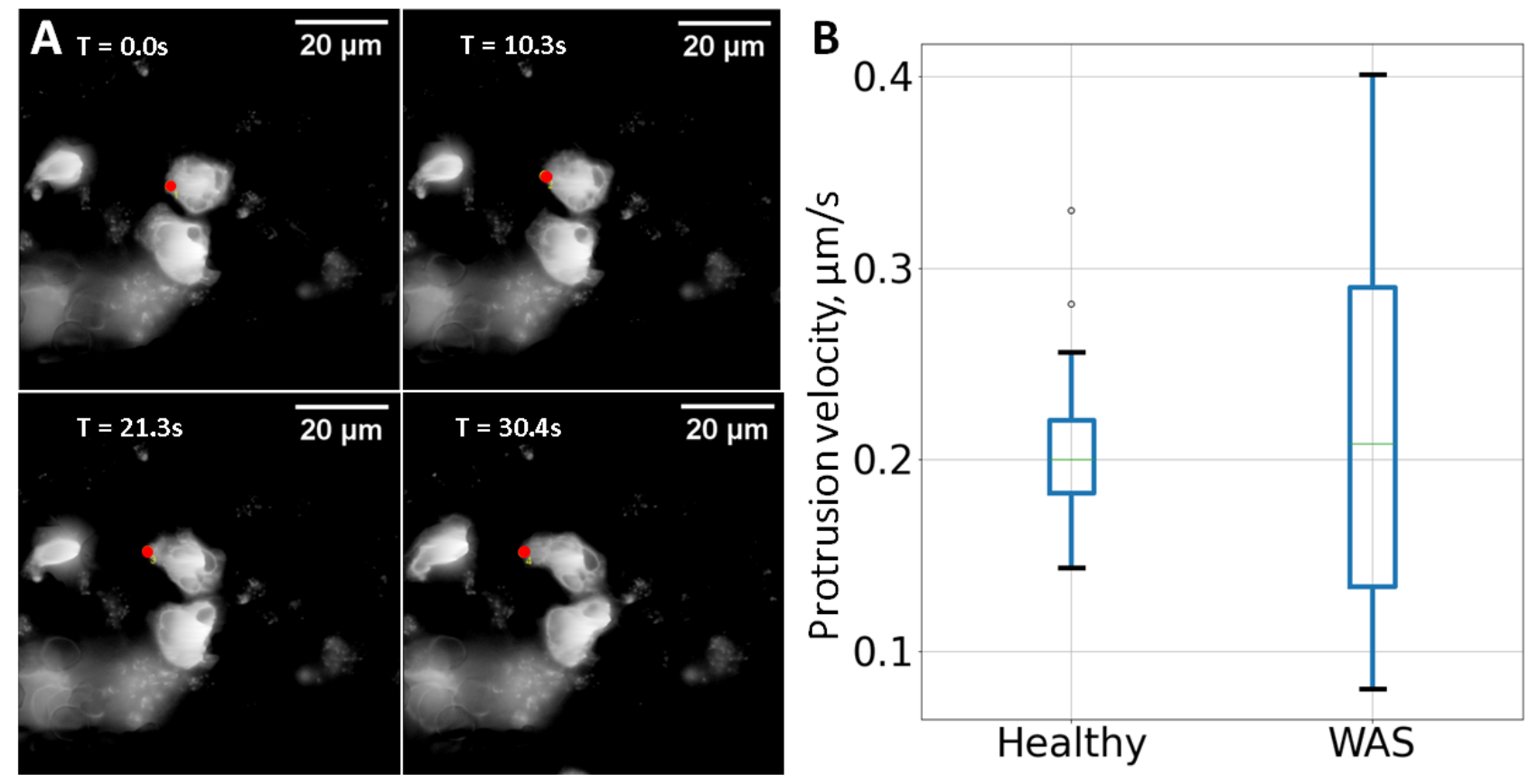

Figure 1. Experimental measurement of the pseudopodium formation rate. Typical images of the growing protrusion. The pseudopodium tips are marked with red circles. (B) Pseudopod velocities for healthy controls and WAS patients. For healthy controls, mean speed of the pseudopod growth was determined to be $0.22 \pm 0.04 \mu \mathrm{m} / \mathrm{s}$. For WAS patients, mean speed of the pseudopod growth was determined to be $0.23 \pm 0.08 \mu \mathrm{m} / \mathrm{s}$. The difference between control and WAS pseudopod growth speed was not significant.

\section{Construction of the computational model of} actin polymerization

To describe the observed experimental data, we have built a minimal stochastic mathematical model of actin cytoskeleton reorganization upon pseudopodium formation. In this model, we have described the following events: the polymerization and depolymerization of F-actin, as well as branching of the growing actin network (Fig. 2A). As the actin polymerization in the pseudopodium occurs only in narrow separate regions on the tips [18], we did not take into account the interaction between different pseudopodia composing the growing protrusion. In the model, the cell plasma membrane was "pushed" by the growing F-actin ends. The critical number of the pushing ends (Nact) was chosen according to experimental data. The probabilities for actin polymerization, branching and depolymerization were estimated from the known reaction rates for human enzymes. Actin exists in two states bound to either ATP or ADP. Monomeric actin hydrolyzes ATP very slowly, and polymeric hydrolyzes ATP fast and irreversibly[19]. The newly assembled part of the filament contains mostly ATP protomers, while older filaments have mainly ADP-protomers[19]. The ADPenriched end (minus or pointed end) of the filament disassembles more readily.

Single actin filaments at steady state add fresh ATP-subunits at the barbed end balanced by dissociation of ADP-actin at the pointed end.

Here, we do not take into account the ADP-actin dissociation because in the present work we are interested only in freshly-assembled actin dynamics in a narrow region near the cell membrane. Typical lengths of actin filaments range from $100 \mathrm{~nm}$ to a few microns [20], and actin polymerization in the model occurs only in a narrow region of leading edge several dozens of $\mathrm{nm}$ wide, so pointed ends are unlikely to get into the area of interest.

In the following equations, $[F]$ denotes the F-actin concentration, $[G]$ is the denotation for G-actin, $k_{o n}$ is the actin polymerization rate constant, $k$ _off is the actin depolymerization rate, $k_{b}$ is the branching rate, [Arp] is the Arp2/3 concentration.

The following equations were used for deriving the G-actin attachment and detachment rates and the possibilities of actin filament branching:

\section{The following equations describe the actin polymerization:}

For actin monomer attachment rate to a filament consisting of $N$ filaments $F_{N}+G \rightarrow F_{(N+1)}$, we have the following equation:

$$
\frac{d[F]}{d t}=k_{o n}[G][F]
$$

Separating the variables, we get:

$$
\frac{d[F]}{[F]}=k_{o n}[G] d t
$$

Integrating the equation above, we get:

$$
\ln \frac{F_{N+1}}{F_{N}}=k_{o n}[G] d t
$$

where $d t$ is the time interval.

$$
\begin{gathered}
\frac{F_{N}}{F_{N+1}}=\exp \left(-k_{o n}[G] d t\right) \\
\frac{\Delta F}{F_{N+1}}=1-\frac{1}{\exp \left(k_{o n}[G] d t\right)}
\end{gathered}
$$


For the possibility of the polymerization, we get the following expression:

$$
P_{\text {on }}=1-\frac{1}{\exp \left(k_{\text {on }}[G] d t\right)}
$$

The possibility of actin depolymerization is acquired in the same fashion:

$$
\begin{gathered}
F_{N}+G \leftarrow F_{(N+1)} \\
P_{o f f}=1-\frac{1}{\exp \left(k_{o f f} d t\right)}
\end{gathered}
$$

2. For the branching reaction, we use a simple approximation to get the expression for the branching possibility:

$$
\begin{aligned}
& F_{N}+\operatorname{Arp} \rightarrow F_{(N+1)} \\
& P_{b}=k_{b}[\operatorname{Arp}] d t
\end{aligned}
$$

The reasons for not picking the simple approximation for polymerization and depolymerization are listed in SI 1.

The polymerization center was assumed to be located at the maximum distance at which F-actin polymerization is possible (Fig. 2B). As it was shown, filopodia can serve as a template for lamellipodia formation[21]. Filopodia are membrane protrusions 60-200 nm in diameter and containing parallel bundles of 10-30 actin filaments[22]. Initially, a subset of 10 F-actin filaments consisting of 2 subunits is generated at the cell membrane. Initial cell diameter is equal to $100 \mathrm{~nm}$.

In each time step, a random number ri from $\mathrm{U}(0,1)$ is generated for every F-actin branch. If $r_{i}$ is smaller than the possibility of F-actin branch growth $\left(P_{o n}\right)$, a single G-actin monomer is added to the branch. In the same fashion, the depolymerization and branching of every branch were modelled. Upon branching, the child branch could start with equal probability both at $70^{\circ} \mathrm{CW}$ or $70^{\circ} \mathrm{CCW}$ in respect to the parent branch direction.

In some cases, the branch growth could be hindered by pressing against the cell membrane. Branch overlap is allowed in this model due to three-dimensipnality of the real lamellipodia and low density $(<7 \% \mathrm{v} / \mathrm{v})$ of the actin mesh in lamellipodia leading to infrequent overlaps [23]. A variant of model with restricted overlap is described in S2 and Fig. $\mathrm{S} 1$. The conclusions drawn from this variant do not differ significantly from the one with allowed branch overlap.

To describe the induction of actin polymerization by phosphoinositides [24], we assume that the actin polymerization occurs only if the tip of the branch is no further than a fixed distance $H$ from the cell membrane (Fig. 2B). Similarly, to take into account the fact that activators of the Arp $2 / 3$ complex, which render Arp2/3 competent for actin filament nucleation, are typically membrane-associated proteins [25], the filaments can branch only if the distance from the cell membrane is lesser than $D$. The number of pushing cell membrane branches, Nact, was set to be
241/ $\mu \mathrm{m}$ [26]. Upon reaching the threshold number of filaments abutting against the membrane, the entire membrane was assumed to move.

The parameter values are given in Table 1.

\begin{tabular}{|c|l|l|l|}
\hline Parameter & Value & Reference & Comment \\
\hline$k_{\text {on }}$ & $7.4\left(\mathrm{~s} \mathrm{x} \mu \mathrm{M}^{-1}\right.$ & {$[27]$} & Actin binding rate \\
\hline$k_{\text {off }}$ & $0.89 \mathrm{~s} \mathrm{~s}^{-1}$ & {$[27]$} & $\begin{array}{l}\text { Actin } \\
\text { depolymerization } \\
\text { rate }\end{array}$ \\
\hline$[$ Arp $]$ & $1.0 \mu \mathrm{M}$ & {$[28]$} & $\begin{array}{l}\text { Arp 2/3 } \\
\text { concentration }\end{array}$ \\
\hline$N$ & 5 & Estimated from [29] & $\begin{array}{l}\text { Initial filament } \\
\text { number }\end{array}$ \\
\hline Nact & $241 / \mu \mathrm{M}$ & {$[30]$} & $\begin{array}{l}\text { Minimal density of } \\
\text { "pushing" } \\
\text { filaments }\end{array}$ \\
\hline
\end{tabular}

Table 1. Model parameters

The branching of the filaments was modelled in the following manner. Taking into account that the Arp2/3 complex is able to bind with four actin monomers corresponding to a couple of beads on the F-actin, we assumed that the new branch can stem from a couple of segments. In the model, each segment can be in either "free" or "occupied" by a growing child branch state (Fig. 2A). Additionally, a filament can grow only if the absolute value of the angle between the normal to the cell membrane direction and the new branch is more than $70^{\circ}$. In other words, the branches must grow forward.

The computational model parameter estimation and validation

The constructed computational model described formation of a network (Fig. 3A). At the first step, we ensured that the growth rate of a single filament is in accordance with the literature data [31]. In the model, the single filament growth rate was 8 subunits per second for the concentration of G-actin of $1 \mu \mathrm{M}$ (data not shown), which corresponds to the reported rate of $1 \mu \mathrm{m} / \mathrm{min}$. Afterwards, a parameter scan was performed to get the values of the parameters $k$, $\mathrm{D}$ and $\mathrm{H}$ which would yield the protrusion velocity closest to the experimental ones. The best fit was obtained for the set of parameter values: $k=9000$ $(M \times S)^{-1}, D=H=7$. In the model the protrusion rate was estimated to be $0.23 \mu \mathrm{m} / \mathrm{s}$. Along the leading edge, the actin filament density is peaked at the center and decreases toward the sides, which came into accordance with experimental data from [32]. Filament density of $1300 \mu \mathrm{m} \mathrm{F-actin} / \mu \mathrm{m}^{3}$ also was in good accordance with experimental data [30].

The adjustment of the computational model to the data on Wiscott-Aldrich syndrome patients

To account for the WASP loss-of-function mutations, we have lowered the model branching rate one order of magnitude and assumed that if WASP localization is disrupted, actin branching and polymerization may occur further from the membrane. In the model, the protrusion growth velocity did not 
A

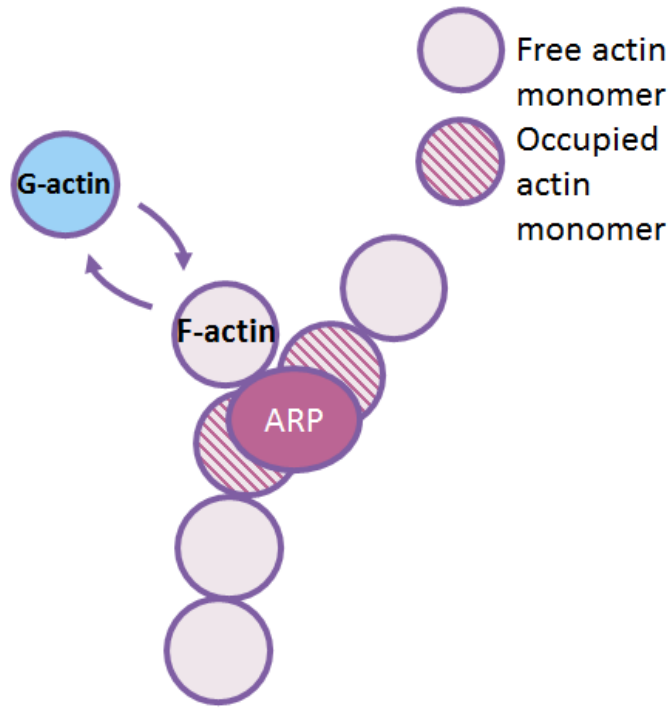

B

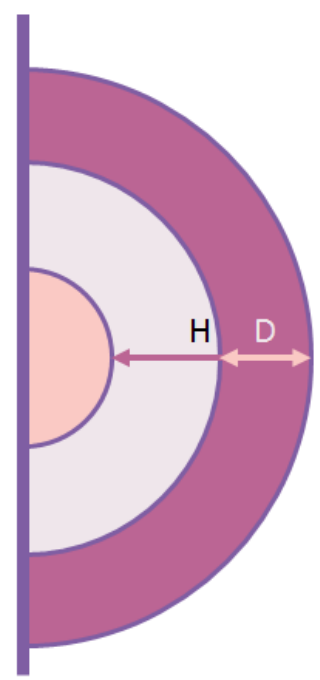

Filament branching zone

Growth zone

No filament growth

Figure 2. Scheme of the computational model. (A) The scheme of stochastic events and species included in the model. A single F-actin filament is assumed to be straight and to be divided into segments. Each segment can be considered to be an actin monomer. New G-actin molecules can attach to and detach from the filament "barbed" end. It is assumed that the child filament begins to grow from the middle between two segments of F-actin at the angle of $70^{\circ}$. If there is a branch growing from the F-actin segments, they are considered occupied and no branching can occur there. (B) The spatial restrictions on the filament growth and branching. The filaments can branch if the distance from the cell membrane is lesser than $D$. Filaments can grow if the distance from the cell membrane is lesser than $H$, where $H>D$.

show any significant dependence on either branching rate $\mathrm{k}$ or branching distance from the membrane $H$ in a large interval of parameter values. For $H>6$, the protrusion velocity did not change more than for $15 \%$. For $k>3000\left(\mathrm{Mxs}^{-1}\right.$, the protrusion velocity did not change more than for $20 \%$. However, a strong dependency was seen on the number of pushing cell membrane branches, Nact (Figure 3D). When Nact was changed from 241 to $482 \mu \mathrm{m} / \mathrm{s}$, lamellipodia growth stopped.

\section{Discussion}

Here, we have studied the dynamics of cellular membrane protrusion growth upon neutrophil motility. We have performed experimental measurements of membrane protrusion growth velocity in healthy donors. The acquired velocity corresponds well with existing data on chemotactic cells such as macrophages [33] or dendritic cells [29]. Additionally, we have measured the linear velocity of the pseudopodia formation for neutrophils of WAS patients (Fig. 1). Surprisingly, the difference between the healthy donors and patients was not significant.

To explain the observed phenomena, we have built a minimal computational model capable of quantitative description of neutrophil membrane protrusion (pseudopodia) formation. The model was similar in design to the mathematical models of Chen [11] and Winkler [26], who constructed models of lamellipodia formation in a generic migrating cell. The molecular machinery included into the model was based on the known data on the regulation of neutrophil pseudopodium formation [34-36]. The correspondence of the membrane protrusion rate predicted by the model to the experimental data indicates the validity of the implied mechanisms.

Although earlier we [3] and others [37] have observed disruption in the movement of WAS patients' neutrophils, the velocity of their protrusions were similar to those of healthy neutrophils. Parameter scan performed here implied that changing of the branching rate or the branching distance in an order of magnitude did not significantly alter the overall protrusion velocity. Together, these data indicate that the chemotaxis disruption in WAS patients could not be explained by the absence of WASP in these patients, which is in general accordance with existing data [38].

However, strong dependence of lamellipodia growth on the number of actin bundles required to push the membrane suggests impaired pseudopod formation as one of the possible mechanisms for cell motility reduction in patients with mutations in forcegenerating protein machinery, for example, MYH9 [39].

\section{Acknowledgements}

The authors are grateful to Ms Daria Morozova (CTP PCP RAS) for the valuable discussions and experimental assistance. 
A

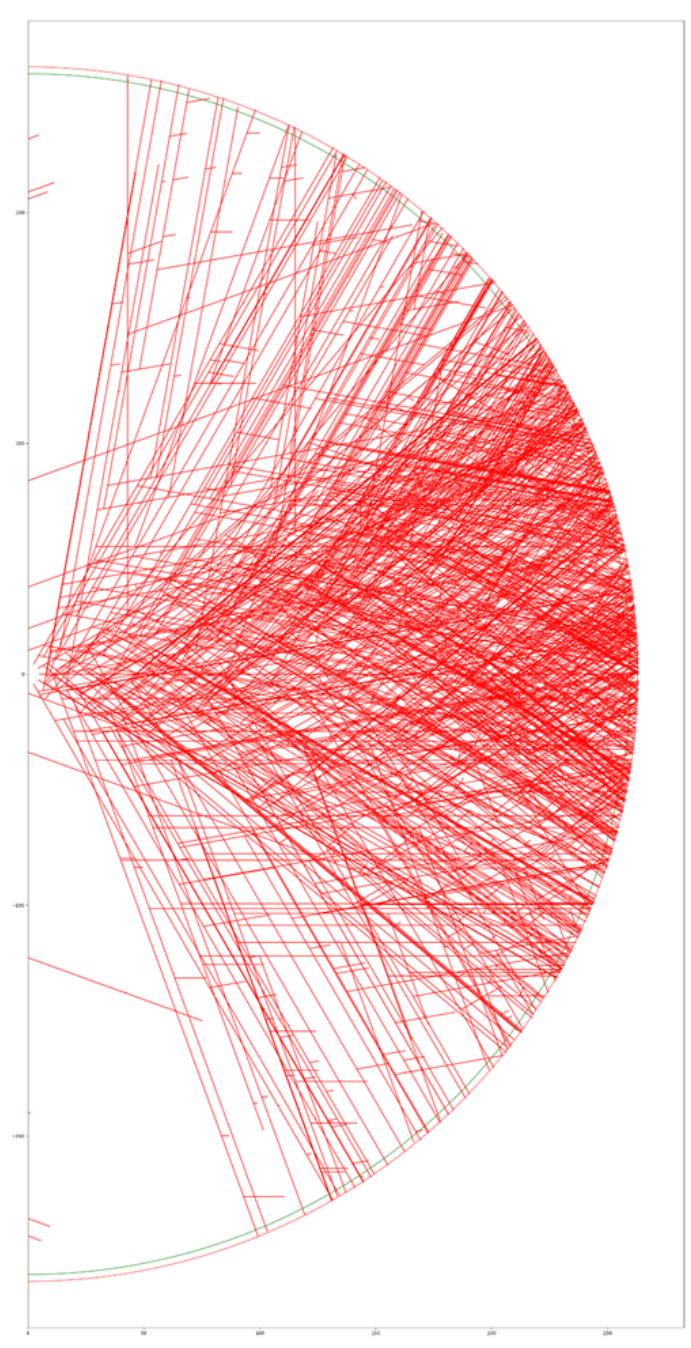

B

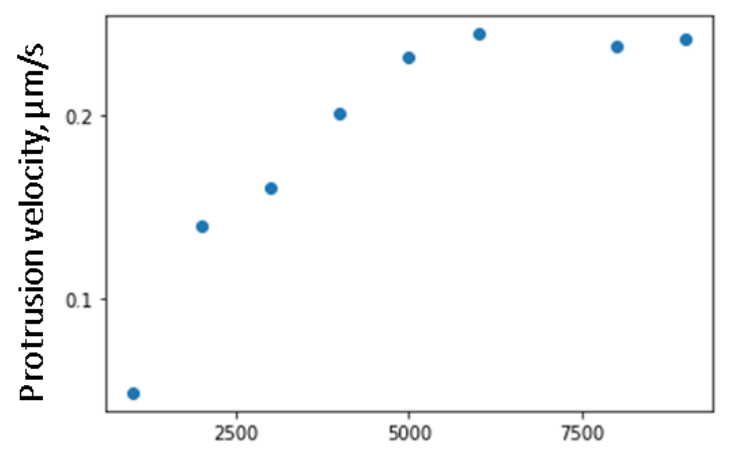

C

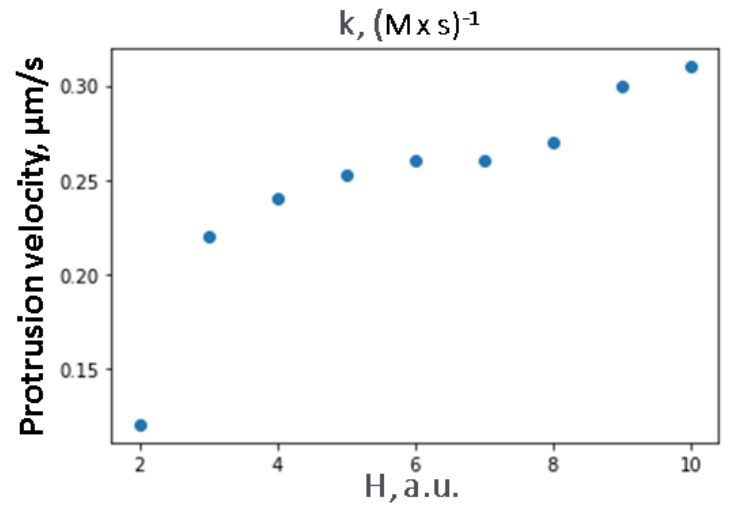

D

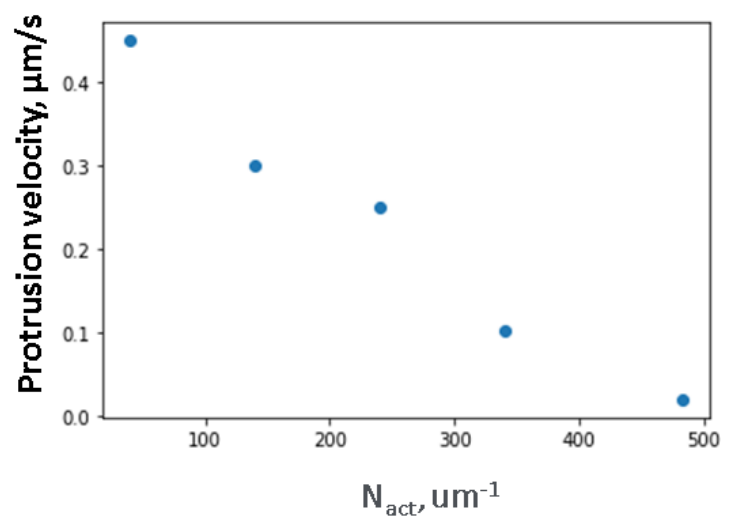

Figure 3. Results of the computational modeling. (A) Typical actin network predicted by the model for parameters $k$ $=9000\left(\mathrm{M} \times \mathrm{S}^{-1}, D=H=7\right.$ actin units. (B) Dependence of the protrusion velocity on the branching rate $\mathrm{k}$. (C) Dependence of the protrusion velocity on the branching distance $H$. (D) Dependence of the protrusion velocity on the number of the actin filaments Nact required to push the membrane.

\section{Funding}

This research was supported by Russian Foundation for Basic Research, grant number 17-0000138 and 21-51-10005 and Lomonosov Moscow State University Digital Medicine School.

\section{References}

1. Puhr-Westerheide D, Schink SJ, Fabritius M, Mittmann L, Hessenauer MET, Pircher J, et al. Neutrophils promote venular thrombosis by shaping the rheological environment for platelet aggregation. Scientific Reports 2019;9:15932. https://doi.org/10.1038/s41598-01952041-8.

2. Szatmary AC, Nossal R, Parent CA, Majumdar R. Modeling neutrophil migration in dynamic chemoattractant gradients: assessing the role of exosomes during signal relay. Molecular Biology of the Cell 2017;28:3457-70. https://doi.org/10.1091/mbc. e17-05-0298.

3. Daria S. Morozova, Alexey A. Martyanov, Sergei I. Obydennyi, Julia-Jessica D. Korobkin, Alexey V. Sokolov, Ekaterina V. Shamova, Irina V. Gorudko, Anna Shcherbina, Mikhail A. Panteleev ANS. Ex vivo observation of granulocyte activity during thrombus formation. BioRxiv 202007131991742020.

4. Pollard TD, Borisy GG. Cellular Motility Driven by Assembly and Disassembly of Actin Filaments. Cell 2003;112:453-65. https://doi.org/10.1016/S00928674(03)00120-X.

5. Ridley AJ. Life at the Leading Edge. Cell 2011;145:101222. https://doi.org/10.1016/j.cell.2011.06.010.

6. Insall RH, Machesky LM. Actin Dynamics at the Leading Edge: From Simple Machinery to Complex Networks. Developmental Cell 2009;17:310-22. https://doi. org/10.1016/j.devcel.2009.08.012.

7. Paul AS, Pollard TD. Review of the mechanism of processive actin filament elongation by formins. Cell Motility and the Cytoskeleton 2009;66:606-17. https:// doi.org/10.1002/cm.20379.

8. Goode BL, Eck MJ. Mechanism and Function of Formins in the Control of Actin Assembly. Annual Review of Biochemistry 2007;76:593-627. https://doi.org/10.1146/ annurev.biochem.75.103004.142647.

9. Zicha, Allen, Brickell, Kinnon, Dunn, Jones, et al. Chemotaxis of macrophages is abolished in the WiskottAldrich syndrome. British Journal of Haematology 1998;101:659-65. https://doi.org/10.1046/j.13652141.1998.00767.x.

10. Rodnick-Smith M, Luan Q, Liu S-L, Nolen BJ. Role and structural mechanism of WASP-triggered conformational 
changes in branched actin filament nucleation by Arp2/3 complex. Proc Natl Acad Sci USA 2016;113:E3834-43. https://doi.org/10.1073/pnas.1517798113.

11. Chen X, Zhu H, Feng X, Li X, Lu Y, Wang Z, et al. Predictive assembling model reveals the self-adaptive elastic properties of lamellipodial actin networks for cell migration. Communications Biology 2020;3:616. https:// doi.org/10.1038/s42003-020-01335-z.

12. Erban R, Flegg MB, Papoian GA. Multiscale Stochastic Reaction-Diffusion Modeling: Application to Actin Dynamics in Filopodia. Bulletin of Mathematical Biology 2014;76:799-818. https://doi.org/10.1007/s11538-0139844-3.

13. Bonilla-Quintana M, Wörgötter F, Tetzlaff $C$, Fauth $M$. Modeling the Shape of Synaptic Spines by Their Actin Dynamics. Frontiers in Synaptic Neuroscience 2020;12. https://doi.org/10.3389/fnsyn.2020.00009.

14. Marée AFM, Jilkine A, Dawes A, Grieneisen VA, Edelstein-Keshet L. Polarization and Movement of Keratocytes: A Multiscale Modelling Approach. Bulletin of Mathematical Biology 2006;68:1169-211. https://doi. org/10.1007/s11538-006-9131-7.

15. Fritz-Laylin LK, Riel-Mehan M, Chen B-C, Lord SJ, Goddard TD, Ferrin TE, et al. Actin-based protrusions of migrating neutrophils are intrinsically lamellar and facilitate direction changes. ELife 2017;6. https://doi. org/10.7554/eLife.26990.

16. Nechipurenko DY, Receveur N, Yakimenko $A O$ Shepelyuk TO, Yakusheva AA, Kerimov RR, et al. Clot Contraction Drives the Translocation of Procoagulant Platelets to Thrombus Surface. Arteriosclerosis, Thrombosis, and Vascular Biology 2019;39:37-47. https://doi.org/10.1161/ATVBAHA.118.311390.

17. Schindelin J, Arganda-Carreras I, Frise E, Kaynig $\mathrm{V}$, Longair M, Pietzsch T, et al. Fiji: an open-source platform for biological-image analysis. Nature Methods 2012;9:676-82. https://doi.org/10.1038/nmeth.2019.

18. Weiner OD, Servant G, Welch MD, Mitchison TJ, Sedat JW, Bourne HR. Spatial control of actin polymerization during neutrophil chemotaxis. Nature Cell Biology 1999;1:75-81. https://doi.org/10.1038/10042.

19. Kudryashov DS, Reisler E. ATP and ADP actin states. Biopolymers 2013;99:245-56. https://doi.org/10.1002/ bip. 22155

20. Kasza KE, Broedersz CP, Koenderink GH, Lin YC, Messner W, Millman EA, et al. Actin Filament Length Tunes Elasticity of Flexibly Cross-Linked Actin Networks. Biophysical Journal 2010;99:1091-100. https://doi.org/10.1016/j.bpj.2010.06.025.

21. Johnson HE, King SJ, Asokan SB, Rotty JD, Bear JE, Haugh JM. F-actin bundles direct the initiation and orientation of lamellipodia through adhesion-based signaling. Journal of Cell Biology 2015;208:443-55. https://doi.org/10.1083/jcb.201406102.

22. Mattila PK, Lappalainen P. Filopodia: molecular architecture and cellular functions. Nature Reviews Molecular Cell Biology 2008;9:446-54. https://doi. org/10.1038/nrm2406.

23. Aroush DR-B, Ofer N, Abu-Shah E, Allard J, Krichevsky O, Mogilner A, et al. Actin turnover in lamellipodial fragments. Curr Biol 2017;27:2963-2973.e14. https:// doi.org/10.1016/j.cub.2017.08.066.

24. Wu C-Y, Lin M-W, Wu D-C, Huang Y-B, Huang H-T, Chen $\mathrm{C}$-L. The role of phosphoinositide-regulated actin reorganization in chemotaxis and cell migration. British Journal of Pharmacology 2014;171:5541-54. https:// doi.org/10.1111/bph.12777.

25. Suetsugu S. Activation of nucleation promoting factors for directional actin filament elongation: Allosteric regulation and multimerization on the membrane. Seminars in Cell
\& Developmental Biology 2013;24:267-71. https://doi. org/10.1016/j.semcdb.2013.01.006.

26. Schmeiser C, Winkler C. The flatness of Lamellipodia explained by the interaction between actin dynamics and membrane deformation. Journal of Theoretical Biology 2015;380:144-55. https://doi.org/10.1016/j. jtbi.2015.05.010.

27. Kuhn JR, Pollard TD. Real-Time Measurements of Actin Filament Polymerization by Total Internal Reflection Fluorescence Microscopy. Biophysical Journal 2005;88:1387-402. https://doi.org/10.1529/ biophysj.104.047399.

28. Mullins RD, Heuser JA, Pollard TD. The interaction of Arp2/3 complex with actin: Nucleation, high affinity pointed end capping, and formation of branching networks of filaments. Proceedings of the National Academy of Sciences 1998;95:6181-6. https://doi. org/10.1073/pnas.95.11.6181.

29. Joosten $B$, Willemse $M$, Fransen J, Cambi A, van den Dries K. Super-Resolution Correlative Light and Electron Microscopy (SR-CLEM) Reveals Novel Ultrastructural Insights Into Dendritic Cell Podosomes. Frontiers in Immunology 2018;9. https://doi.org/10.3389/ fimmu.2018.01908.

30. Abraham VC, Krishnamurthi V, Taylor DL, Lanni F. The Actin-Based Nanomachine at the Leading Edge of Migrating Cells. Biophysical Journal 1999;77:1721-32. https://doi.org/10.1016/S0006-3495(99)77018-9.

31. Crevenna AH, Naredi-Rainer N, Schönichen A, Dzubiella J, Barber DL, Lamb DC, et al. Electrostatics Control Actin Filament Nucleation and Elongation Kinetics. Journal of Biological Chemistry 2013;288:12102-13. https://doi.org/10.1074/jbc.M113.456327.

32. Ofer $\mathrm{N}$, Mogilner A, Keren K. Actin disassembly clock determines shape and speed of lamellipodial fragments. Proceedings of the National Academy of Sciences 2011;108:20394-9. https://doi.org/10.1073/ pnas. 1105333108.

33. Kapellos TS, Taylor L, Lee H, Cowley SA, James WS, Iqbal AJ, et al. A novel real time imaging platform to quantify macrophage phagocytosis. Biochemical Pharmacology 2016;116:107-19. https://doi. org/10.1016/j.bcp.2016.07.011.

34. Mócsai $A$, Walzog B, Lowell CA. Intracellular signalling during neutrophil recruitment. Cardiovascular Research 2015;107:373-85. https://doi.org/10.1093/cvr/cvv159.

35. Wong K, Pertz O, Hahn K, Bourne H. Neutrophil polarization: Spatiotemporal dynamics of RhoA activity support a self-organizing mechanism. Proceedings of the National Academy of Sciences 2006;103:3639-44. https://doi.org/10.1073/pnas.0600092103.

36. Ku C-J, Wang Y, Weiner OD, Altschuler SJ, Wu LF. Network Crosstalk Dynamically Changes during Neutrophil Polarization. Cell 2012;149:1073-83. https:// doi.org/10.1016/

37. Ochs H, Slichter S, Harker L, Von Behrens W, Clark $\mathrm{R}$, Wedgwood R. The Wiskott-Aldrich syndrome: studies of lymphocytes, granulocytes, and platelets. Blood 1980;55:243-52. https://doi.org/10.1182/blood. V55.2.243.243.

38. Blundell MP, Worth A, Bouma G, Thrasher AJ. The Wiskott-Aldrich syndrome: The actin cytoskeleton and immune cell function. Disease Markers 2010;29:15775. https://doi.org/10.3233/DMA-2010-0735.

39. Zehrer A, Pick R, Salvermoser M, Boda A, Miller M, Stark K, et al. A Fundamental Role of Myh9 for Neutrophil Migration in Innate Immunity. The Journal of Immunology 2018;201:1748-64. https://doi. org/10.4049/jimmunol.1701400. 